\title{
Volatile memory acquisition using backup for forensic investigation
}

\begin{abstract}
Nowadays mobile phones are used all over the world for the communication purposes. The capabilities of these devices are improved during the past few years. Due to their capabilities, mobile devices are used broadly in criminal activities especially in cybercrime. The volatile data stored in mobile phones usually contain important evidences regarding the crime. However, collecting these volatile data in a forensically sound manner would not be easy. This paper proposes a new approach for acquiring the volatile data inside a mobile phone in a forensically sound manner that minimizes the chance of evidence modification or lost.
\end{abstract}

Keyword: Mobile phone forensics; Mobile phone investigation; Digital forensics; Volatile data; Backup 